\title{
Amplitude and lifetime of radial modes in red giant star spectra observed by Kepler ${ }^{\star}$
}

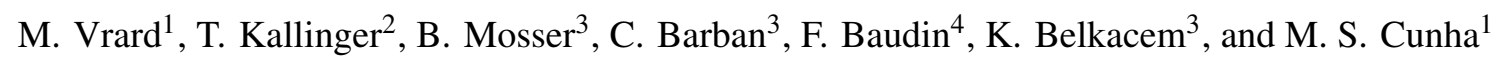 \\ ${ }^{1}$ Instituto de Astrofísica e Ciências do Espaço, Universidade do Porto, CAUP, Rua das Estrelas, 4150-762 Porto, Portugal \\ e-mail: mathieu.vrard@astro.up.pt \\ 2 Institute for Astrophysics (IfA), University of Vienna, Türkenschanzstrasse 17, 1180 Vienna, Austria \\ 3 LESIA, CNRS, PSL Research University, Université Pierre et Marie Curie, Université Denis Diderot, Observatoire de Paris, \\ 92195 Meudon Cedex, France \\ ${ }^{4}$ Université Paris-Sud, CNRS, Institut d'Astrophysique Spatiale, UMR 8617, 91405 Orsay Cedex, France
}

Received 16 December 2017 / Accepted 8 May 2018

\begin{abstract}
Context. The space-borne missions CoRoT and Kepler have provided photometric observations of unprecedented quality. The study of solar-like oscillations observed in red giant stars by these satellites allows a better understanding of the different physical processes occurring in their interiors. In particular, the study of the mode excitation and damping is a promising way to improve our understanding of stellar physics that has, so far, been performed only on a limited number of targets.

Aims. The recent asteroseismic characterization of the evolutionary status for a large number of red giants allows us to study the physical processes acting in the interior of red giants and how they are modified during stellar evolution. In this work, we aim to obtain information on the excitation and damping of pressure modes through the measurement of the stars' pressure mode widths and amplitudes and to analyze how they are modified with stellar evolution. The objective is to bring observational constraints on the modeling of the physical processes behind mode excitation and damping.

Methods. We fit the frequency spectra of red giants with well-defined evolutionary status using Lorentzian functions to derive the pressure mode widths and amplitudes. To strengthen our conclusions, we used two different fitting techniques.

Results. Pressure mode widths and amplitudes were determined for more than 5000 red giants. With a stellar sample two orders of magnitude larger than previous results, we confirmed that the mode width depends on stellar evolution and varies with stellar effective temperature. In addition, we discovered that the mode width depends on stellar mass. We also confirmed observationally the influence of the stellar metallicity on the mode amplitudes, as predicted by models.
\end{abstract}

Key words. asteroseismology - convection - stars: solar-type - stars: evolution - stars: interiors - methods: data analysis

\section{Introduction}

The launches of the space missions MOST (Walker et al. 2003), CoRoT (Baglin et al. 2006), and Kepler (Borucki et al. 2010) have revolutionized the field of asteroseismology and especially the study of solar-like pulsators. This is particularly the case for red giant stars, MOST and CoRoT having revealed the wealth of asteroseismic observations for this kind of star (e.g., Kallinger et al. 2008; De Ridder et al. 2009), before being confirmed by Kepler (e.g., Bedding et al. 2010). Their spectra show a complicated pattern, exhibiting radial pressure modes as well as nonradial mixed modes. In cool stars with a convective envelope, pressure modes are the signature of acoustic waves excited by turbulent convection in the outer stellar layers. They carry information on the internal structure of the envelope and on the different physical processes occurring inside these objects. Their spectra follow the so-called universal red giant oscillation pattern (Mosser et al. 2011b), which describes the regularity of the pressure mode pattern characterized by two quantities: the frequency of maximum oscillation power $\left(v_{\max }\right)$ and the mean frequency difference $(\Delta v)$ between consecutive pressure modes of

\footnotetext{
* The full results are only available at the CDS via anonymous ftp to cdsarc.u-strasbg.fr $(130.79 .128 .5)$ or via http://cdsarc.u-strasbg.fr/viz-bin/qcat?J/A+A/616/A94
}

the same angular degree. In combination with effective temperature $\left(T_{\text {eff }}\right)$, these global seismic parameters are used to precisely constrain the stellar mass and radius through scaling relations (e.g., Kallinger et al. 2010a).

Mixed modes, first noticed in red giants by Bedding et al. (2010) and then identified by Beck et al. (2011), are the signature of waves behaving as acoustic waves in the stellar envelope and as gravity waves in the core. Therefore, they carry unique information on the red giant core properties, which allows us to distinguish between core-helium burning red giants (clump stars) and hydrogen-shell burning red giants (RGB stars; Bedding et al. 2011; Mosser et al. 2011a, 2012b). The identification of the stars evolutionary status was then performed on a large number of objects (Kallinger et al. 2012; Stello et al. 2013; Elsworth et al. 2017; Hon et al. 2017). Recent advances (Mosser et al. 2014, 2017; Vrard et al. 2016) make it even possible to precisely follow the evolution of the stellar core mass during the red giant phase.

Among the physical processes occurring inside red giants, those responsible for mode excitation and damping are most difficult to characterize since they involve non-adiabatic physical processes. Constraints on the excitation and damping mechanisms can be obtained by measuring the mode amplitudes and lifetimes, the latter being directly related to the mode widths. The physical mechanisms behind mode damping are 
still poorly identified. Several studies were conducted to characterize the dominant one in solar-like pulsators, leading to different conclusions (e.g., Goldreich \& Kumar 1991; Balmforth 1992; Dupret et al. 2006). The absence of a global agreement on this subject makes theoretical predictions difficult. A first attempt to characterize the scaling relations between pressure mode damping and stellar parameters, such as the effective temperature, was done by Chaplin et al. (2009), but these theoretical predictions were later challenged by observations (Baudin et al. 2011; Houdek 2012). However, recent theoretical studies taking the latest observational results into account seem to point towards the perturbation of turbulent pressure being the main physical mechanism behind mode damping in solar-like pulsators (Belkacem et al. 2012; Houdek et al. 2017). Several other studies intended to constrain the mode damping based on the observed mode lifetimes, but they are mainly focused on main sequence and subgiants stars (Appourchaux et al. 2012, 2014; Lund et al. 2017). Few studies have been conducted for red giants and they were restricted to a limited number of targets (Baudin et al. 2011; Corsaro et al. 2012, 2015; Handberg et al. 2017). A thorough measurement of the pressure mode lifetime for a large number of red giants is still to be performed. The physical mechanism responsible for pressure mode excitation, better understood than the damping, has been identified as the Reynolds stresses, which are induced by turbulent convection (e.g., Balmforth 1992; Belkacem et al. 2006).

Several studies propose scaling relations between mode amplitudes and stellar parameters (e.g., Kjeldsen \& Bedding 1995, 2011; Houdek et al. 1999; Samadi et al. 2007). A scaling relation with mode amplitude varying as $(L / M)^{0.7}$ was predicted for main sequence stars, where $M$ is the mass of the star and $L$ its luminosity. Kjeldsen \& Bedding (2011) introduced afterward the fact that the relative variation of the luminosity not only scales with $L / M$, but also with the mean lifetime of the modes and with the large separation. It was later extended to red giant stars by Samadi et al. (2012). This relation has been tested on red giants in numerous studies (Mosser et al. 2010, 2012a; Huber et al. 2010; Baudin et al. 2011; Corsaro et al. 2013; Kallinger et al. 2014), which found scalings varying typically as $L^{0.8} / M^{1.2}$. This difference compared to a scaling as a power law of $L / M$ indicates the relevance of examining the role of an additional term such as mode lifetimes in the mode amplitude.

Here, we analyze the pressure mode amplitudes and lifetimes in a large number of red giant oscillation spectra. Using several thousand oscillation spectra from the main Kepler mission, we aim to investigate the dependence between the mode parameters and the global stellar parameters and follow their variation with the stellar evolution. We then compare our results with previous measurements and theoretical predictions. The layout of the paper is as follows. In Sect. 2, we recall how the observed mode width and amplitude are linked with the mode damping and excitation. In Sect. 3, we describe the two fitting methods that we used. The analysis of the measured mode widths and amplitudes is conducted in Sects. 4 and 5, respectively. Section 6 is devoted to conclusions.

\section{Observational constraints on mode damping and excitation}

\subsection{Mode damping}

Mode widths $\Gamma$, defined as the mode full width at half maximum (FWHM), are directly related to mode lifetime $\tau$ and, consequently, to the considered mode damping rate $\eta$ following the relation (e.g., Samadi et al. 2015)
$\Gamma=\frac{1}{\pi \tau}=\frac{\eta}{\pi}$

Measurements of the mode width therefore directly constrain the mode damping.

\subsection{Mode amplitude}

The radial mode amplitude is related to the energy injected into the modes and can therefore be linked to the local mean squared velocity variation $(v)$ of the oscillations (see, e.g., Eq. (4.15) of Samadi et al. 2015), which is given by (e.g., Dupret et al. 2009; Samadi et al. 2013)

$v^{2}=\frac{P}{2 \eta M I}$

where $P$ is the time-averaged power stochastically supplied to the mode and $I$ is the mode inertia. The mode amplitude is then linked to the $P$ parameter and the mode damping.

\section{Fitting procedure}

Our aim is to assess the dependence of the pressure mode widths and amplitudes on the stellar fundamental parameters. To achieve this, we use two different automated methods. The first is based on a frequentist fitting and the second follows a Bayesian approach. The independent development of these methods allows us to strengthen the conclusions regarding the different discovered dependencies.

\subsection{Data}

Long-cadence data from Kepler up to the quarter Q17 are used, which correspond to 44 months of photometric observations. The red giant sample was selected based on the APOKASC catalog (Pinsonneault et al. 2014), which resulted from an asteroseismic and spectroscopic joint survey of Kepler targets. This survey includes about 6300 red giants. In the following, we focus our analysis on a subset of 5523 stars with a signal-to-noise ratio $(\mathrm{S} / \mathrm{N})$ sufficient to determine their evolutionary states using the following techniques.

The evolutionary status is determined for each star using the method of Kallinger et al. (2012), which is based on the evaluation of the difference between clump and RGB stars in their pressure mode pattern. This method is completed, when possible, by the one of Vrard et al. (2016), which uses the mixed-mode pattern to distinguish clump and RGB stars.

As stated above, the spectra of red giant stars exhibit pressure modes as well as mixed modes. Because of the nature of gravity waves, radial modes $(\ell=0)$ are not affected by the coupling between gravity and pressure waves, contrary to higher angular degree modes, and they are not split by rotation. We therefore focus our study on radial modes since they are the only pure pressure modes present in the red giant oscillation spectra.

\subsection{General description}

The two mode-fitting methods show important similarities in their execution, with four main steps.

- Determination of the seismic global parameters $(\Delta v$ and $\left.v_{\max }\right)$ as well as the characterization of the granulation background. 
- Refinement of the estimate of the large separation through the use of the pressure mode frequency positions. These modes indeed follow a precisely described pattern that has been characterized in previous works (e.g., Kallinger et al. 2010b; Mosser et al. 2011b). The use of this pattern allows a precise adjustment of $\Delta v$ and to obtain an approximation of the position of the radial modes.

- Identification of the radial modes in the spectra with an estimation of their frequency positions.

- Fitting of the radial and nearby modes on top of the previously estimated background by Lorentzian profiles, (one per mode) which are defined by

$$
\mathcal{L}(v)=\sum_{k} \frac{A_{k}^{2} /\left(\pi \Gamma_{k}\right)}{1+\left(\frac{2\left(v-v_{k}\right)}{\Gamma_{k}}\right)^{2}},
$$

where $A_{k}$ is the amplitude of the $k$ th mode, $v_{k}$ is its oscillation mode frequency, and $\Gamma_{k}$ is its mode linewidth (FWHM, see Eq. (1)). The radial mode widths, heights, amplitudes and frequencies are then extracted for all significant modes.

\subsection{First method: frequentist approach (FREQ)}

The method FREQ realizes the above mentioned steps as follows.

The first step is performed by the use of the envelope autocorrelation function as described in Mosser \& Appourchaux (2009). It allows, for a given spectrum, the determination of a first estimate of $\Delta v$ and $v_{\max }$ as well as the background component parameterized as a power law (Eq. (3) of Mosser et al. 2012a).

The second step is carried out by means of the universal pattern as described in Mosser et al. (2011b), which refines the value of $\Delta v$ from the autocorrelation function and allows us to estimate the frequency position of the radial modes.

The third step is realized by smoothing the power density spectrum (PDS) with a low-pass filter with a width equal to $\Delta v / 100$ and locating the local maximas within one tenth of $\Delta v$ around the estimated radial mode positions. These local peaks are considered to be significant if their heights exceed a threshold corresponding to the rejection of the pure noise hypothesis with a confidence level of $99.9 \%$. Among those peaks, the $\ell=0$ and $\ell=2$ doublets can easily be identified as the modes with the highest mode heights and largest mode widths. If there are more than two peaks exhibiting a height and width value higher than half the height and half the width of the highest and widest peak, respectively, then the radial mode is identified as the one closest to the frequency corresponding to the first estimation of the radial mode frequency position.

The last step is performed by fitting the radial and the nearby modes with Lorentzian profiles (Fig. 1) using the maximum likelihood estimator technique described in Toutain \& Appourchaux (1994) and Appourchaux (2014). The fit consists of a background component parameterized as a power law (Eq. (3) of Mosser et al. 2012a) and several Lorentzian profiles, one for each mode (see Eq. (3)), following the model of Barban et al. (2010) and Vrard et al. (2015). This allows us to extract the frequency, height, width, and amplitude of all significant modes, including radial and quadrupolar modes. The use of a power law as a model for the background has already been proven to be acceptable for small portions of the spectrum (Mosser et al. 2012a).

We use this method on the Kepler data previously described but select only the stars for which we can extract at least three

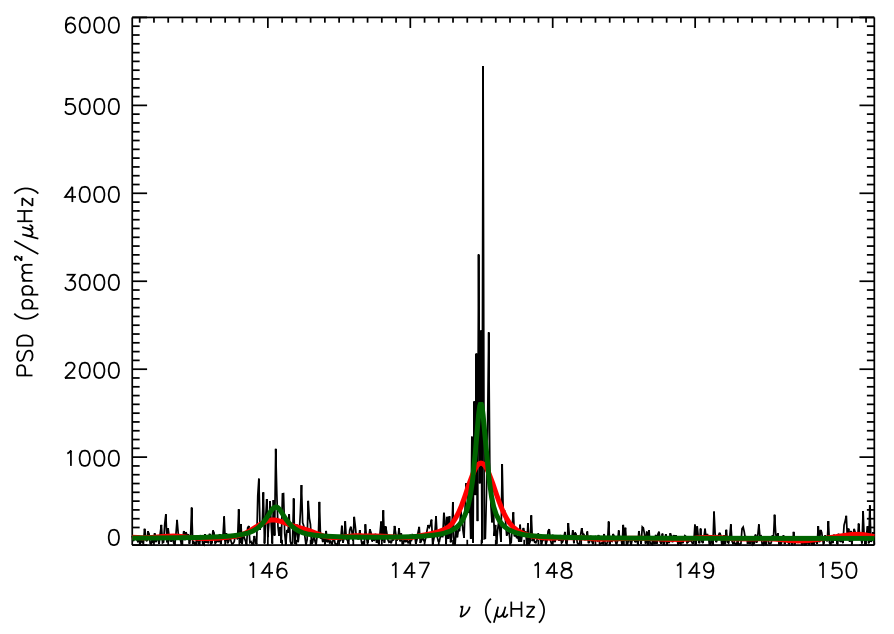

Fig. 1. Power density spectrum for the star KIC 3758505 (black line) and the range of frequencies corresponding to one tenth of $\Delta v$. The red line represents the smoothed spectrum. Our best fit (green line) consists of two Lorentzians and a power law background component with the peaks at about 147.5 and $146 \mu \mathrm{Hz}$ being a radial and $\ell=2$ mode, respectively.

radial modes with uncertainties on their measured parameters lower than $10 \%$. This criterion is satisfied by 5221 stars.

Since the method FREQ is fully automated, spurious measurements are possible. It sometimes happens that a radial mode has a lower amplitude than its nearby $\ell=2$ mode. If so, confusion in the mode identification is possible and the $\ell=2$ mode is identified as a radial mode. Such incidences are, however, quite rare since the quadrupole modes typically have an amplitude two times lower than the radial modes (Mosser et al. 2012a). Following on the different tests we performed, we confirm that this kind of confusion happens in less than $1 \%$ of all investigated stars.

Several previous studies also pointed out that there is a significant impact from the background parameterization on the mode fitting and therefore on the mode widths and amplitudes (e.g., Appourchaux et al. 2014). However, it was previously demonstrated that the use of a power law as a background model offers enough precision for estimating the background in the limited frequency range where oscillations are observed (Mosser et al. 2012a). Nevertheless, we cannot rule out the existence of global systematic effects connected to the selection of this particular background model.

The complete data sets listing the mode-fitting results that were obtained with this method for each star are available at the CDS (see Appendix A).

\subsection{Second method: probabilistic approach (PROB)}

For the second approach, the first step is done by using the approach of Kallinger et al. (2014) that characterizes the granulation background in the vicinity of the oscillation power excess and models the global shape of the PDS with the superposition of two super-Lorentzian functions ${ }^{1}$ and a Gaussian centered on $v_{\max }$, where the latter serves as a proxy for the oscillation power.

The second and third steps are performed by means of a fit based on the following relation, which mimics the second-order asymptotic expansion

\footnotetext{
1 Super-Lorentzian functions are similar to Lorentzians but with a different exponent in their denominator (see e.g., Kallinger et al. 2014).
} 
Table 1. Global seismic parameters.

\begin{tabular}{lcccccccc}
\hline \hline KIC number & $\Delta v(\mu \mathrm{Hz})$ & $v_{\max }(\mu \mathrm{Hz})$ & $\Gamma_{0}\left(v_{\max }\right)(\mu \mathrm{Hz})$ & $\delta \Gamma_{0}\left(v_{\max }\right)(\mu \mathrm{Hz})$ & $A_{0, \text { bol }}(\mathrm{ppm})$ & $M / M_{\odot}$ & $\delta M_{/} M_{\odot}$ & Evolution \\
\hline 1027337 & 6.95 & 75.1 & 0.099 & 0.0111 & 60.53 & 1.49 & 0.044 & 1 \\
1160789 & 3.61 & 25.3 & 0.156 & 0.0301 & 145.66 & 0.79 & 0.023 & 2 \\
1161447 & 4.22 & 36.8 & 0.158 & 0.0274 & 74.28 & 1.34 & 0.039 & 2 \\
1161618 & 4.11 & 33.9 & 0.139 & 0.0239 & 111.61 & 1.14 & 0.033 & 2 \\
\hline
\end{tabular}

Notes. Seismic parameters, as described in the text, of four stars among the 5523 that were considered in this study. The full table for the whole data set is available at CDS. The columns correspond to, from left to right, the star KIC number, its large separation $(\Delta v)$, its frequency of maximum oscillation power $\left(v_{\max }\right)$, its global radial mode width $\left(\Gamma_{0}\left(v_{\max }\right)\right)$, the $1 \sigma$ uncertainties on this parameter, its bolometric mode amplitude $A_{0, \text { bol }}$, its stellar mass $(M)$ and the $1 \sigma$ uncertainties on this parameter. The "evolution" value on the last column provides the evolutionary stage: 1 for RGB stars and 2 for clump stars.

$v_{n, \ell}=v_{c}+\Delta v\left[n-n_{c}+\frac{\alpha}{2}\left(n-n_{c}\right)^{2}\right]-\delta v_{0 \ell}$,

where $v_{c}$ is the frequency of the radial mode closest to $v_{\max }, n_{c}$ is its radial order, $\delta v_{0 \ell}$ is the small separation and $\alpha$ a curvature term. This relation provides relevant estimates for $v_{c}, \Delta v$, and the other parameters and allows a precise estimation of the position of the pure pressure $\ell=0$ and 2 modes in five radial orders around $v_{\max }$. Tests have shown that we can re-construct the full observable $\ell=0$ and 2 mode pattern of red giants in all evolutionary stages covered by the Kepler observations to better than about $2 \%$ of $\Delta v$.

The last step consists in fitting the individual radial modes with Lorentzian profiles and rating their statistical significance. To do so, we fit the sum of two Lorentzian profiles, following Eq. (3), on top of the previously defined background to the PDS in the frequency range of -1.5 to +0.5 times $\delta v_{02}$ around the estimated position of a given radial mode. For the fit we use the Bayesian nested sampling algorithm MultiNest (Feroz et al. 2009), which delivers posterior probability distributions for the frequency $\left(v_{k}\right)$, rms amplitude $\left(A_{k}\right)$, and width $\left(\Gamma_{k}\right)$ of the $k$ th profile, from which we determine the bestfit parameters and their $1 \sigma$-uncertainty. A problem in this approach is the potential presence of mixed $\ell=1$ modes in the close vicinity of the radial or quadrupole mode, which would distort the fit. This is tested by dividing the PDS by the initial fit, which then represents the residuals in terms of a height-to-background ratio (HBR). If we find sharp peaks exceeding a HBR of eight, they are eliminated from the PDS and the fit is redone.

To rate the significance of the fitted modes, we compare the above fit to fits including only one Lorentzian profile (i.e., only the $\ell=0$ or $\ell=2$ mode is detectable in the given frequency range) and no profile at all (i.e., only an average value). A scenario is then considered to be statistical significant if its model evidence (as delivered by MuLTINEST) is at least ten times ${ }^{2}$ larger than the combined model evidence of the other scenarios. We repeat this procedure for $\ell=0$ and 2 doublets for relative radial orders of up to \pm 5 , which gives in the best case a sequence of 11 radial modes, for which we can determine their frequencies within typically $5-10 \mathrm{nHz}$ in the center of the power excess and some $10 \mathrm{nHz}$ at the wings. The median relative uncertainty of the mode width in the central region of the power excess is about $12 \%$.

The method PROB represents a reliable approach to determine the radial mode parameters of red giants in a fully automatic way. Extensive tests have shown that the misidentification

\footnotetext{
2 In probability theory an odds ratio of 10:1 is considered as strong evidence (Jeffreys 1998).
}

of a mode is very rare. It is, however, quite expensive in terms of computation time (about 15 min for a single star on a personal computer). The complete data sets listing the mode-fitting results from this method will be published in a forthcoming paper (Kallinger et al. in prep.).

\subsection{Computation of the global radial mode width and the bolometric amplitude}

For each star and each method, the global radial mode width $\Gamma_{0}\left(v_{\max }\right)$ is computed as the weighted mean of the three pressure modes closest to $v_{\max }$, where we use the mode amplitude as weight. Such a definition minimizes systematic effects due to the possible spurious measurements previously mentioned. Moreover, at these frequencies, the observed mode widths are expected to exhibit almost identical values (Houdek et al. 1999; Appourchaux et al. 2014). $\Gamma_{0}\left(v_{\max }\right)$ then corresponds to the nearly uniform mode widths, which are observed close to $v_{\max }$.

Concerning the mode amplitude, we first determine the average maximum mode amplitude $A_{0, \max }$ for a given star as the mean amplitude of the three radial modes closest to $v_{\max }$, following Baudin et al. (2011). Before analyzing the amplitude variations, we bolometrically correct the Kepler observations according to

$A_{0, \text { bol }}=A_{0, \max }\left(\frac{T_{\text {eff }}}{T_{\mathrm{K}}}\right)^{0.80}$,

with $T_{\mathrm{K}}=5934 \mathrm{~K}$ (Ballot et al. 2011). This correction accounts for the wavelength dependence of the photometric variation integrated over the Kepler bandpass. The complete data are available at the CDS (see Table 1).

\subsection{Comparison of the methods}

In order to evaluate the consistency of our results, we compare the mode parameters for the two methods. Figure 2 gives a comparison between the radial mode widths and amplitudes, respectively, determined with the different methods.

The results are consistent with each other for both parameters and their observed dispersion is consistent with the error bars. However, we can see that the FREQ $\Gamma_{0}\left(v_{\max }\right)$ values are globally overestimated by about $10 \%$ with respect to the PROB values. This deviation is independent of the evolutionary stage as well as of the mass of the stars.

In order to more precisely characterize this bias, we perform a linear fit on the different values we found for the two methods. The results are the following: 
M. Vrard et al.: Radial mode amplitude and lifetime in red giants
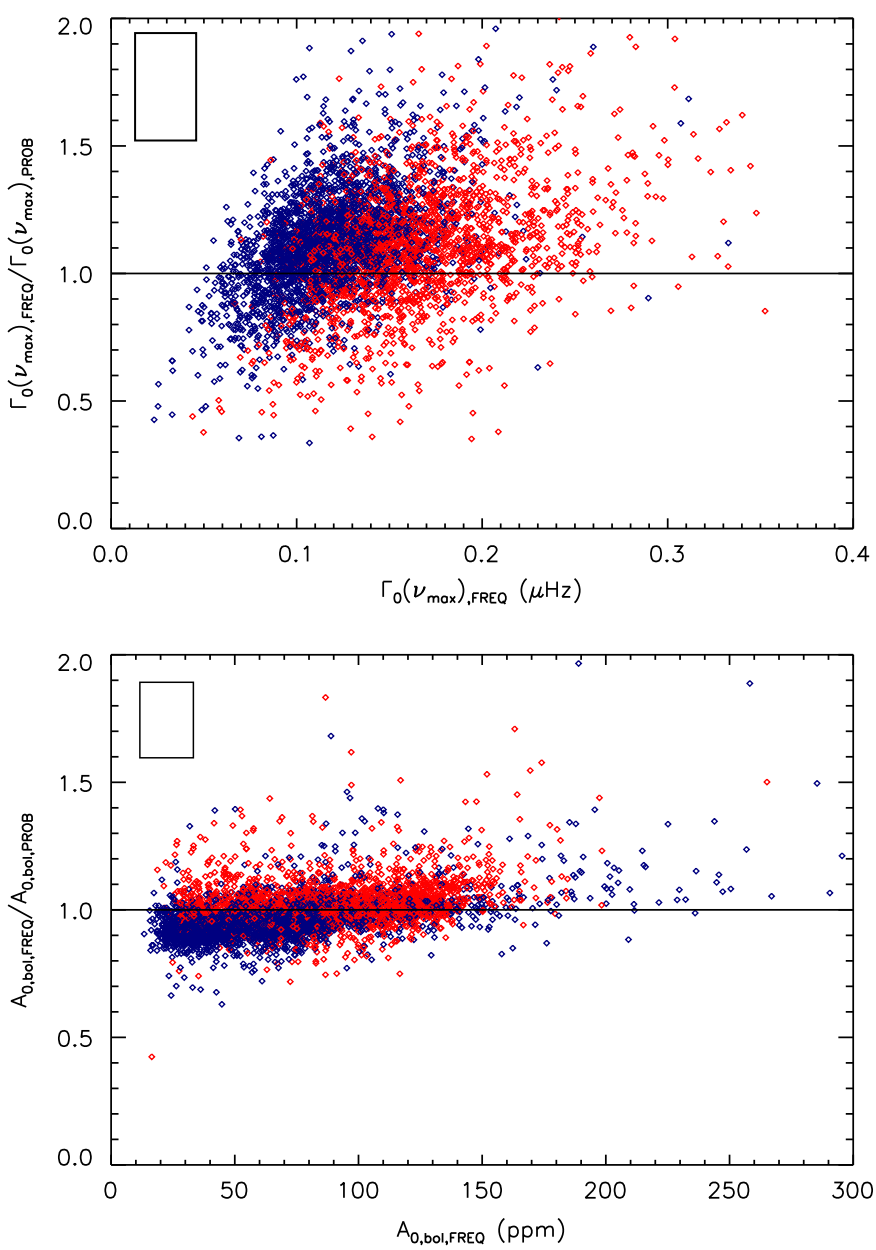

Fig. 2. Top: ratio between the global radial mode widths $\Gamma_{0}\left(v_{\max }\right)$ obtained with the FREQ method and those obtained with the PROB method as a function of the global radial mode width $\Gamma_{0}\left(v_{\max }\right)$ obtained with the FREQ method. Bottom: ratio between the bolometric amplitudes $A_{0 \text { obl }}$ obtained with the FREQ method and those obtained with the PROB method as a function of the bolometric amplitudes $A_{0, \text { bol }}$ obtained with the FREQ method. For both panels, the black line represents the 1:1 relation and the mean uncertainties are given by the black rectangle in the upper left corner. The red diamond and blue triangle symbols indicate clump and RGB stars, respectively.

$$
\left\{\begin{array}{l}
\Gamma_{0}\left(v_{\max }\right)_{, \mathrm{FREQ}}=(0.91 \pm 0.02) \Gamma_{0}\left(v_{\max }\right)_{, \mathrm{PROB}} \\
+0.023 \pm 0.002, \\
A_{0, \text { bol }}, \mathrm{FREQ}=(1.04 \pm 0.02) A_{0, \mathrm{bol}}, \mathrm{PROB}-3.8 \pm 0.35 .
\end{array}\right.
$$

The slopes we find for the fits are close to one for both parameters, indicating that the results of the two methods scale similarly with the parameters under consideration. The small slope for $\Gamma_{0}\left(v_{\max }\right)$ could indicate the existence of a small trend, but the measured values being concentrated in a small range of $\Gamma_{0}\left(v_{\max }\right)$, does not allow firm conclusions to be drawn. Nevertheless, in the case of mode widths, the constant in the fit corresponds to about $10 \%$ of the line width values, reflecting the bias towards larger values of the line widths obtained with the FREQ method.

In order to estimate the impact of the mode width bias, we compute the mean quadratic deviations between the $\Gamma_{0}\left(v_{\max }\right)$ of the two methods. We find that the value of this parameter corresponds to $0.036 \mu \mathrm{Hz}$, which is smaller than the mean uncertainty on the mode width $(0.042 \mu \mathrm{Hz})$. We therefore conclude that this bias does not significantly impact the further interpretation of the results.

\section{Mode widths}

In this section, we show the results we obtain for the radial mode widths, compare them to theoretical predictions and previous results on red giants, and discuss the implications.

\section{1. $\Gamma_{0}\left(v_{\max }\right)$ variation with the stellar evolution: evidence for a mass dependence}

Since the large separation is directly related to the mean stellar density, we can use $\Delta v$ to estimate the variation of $\Gamma_{0}\left(v_{\max }\right)$ with stellar evolution along the RGB. Moreover, we can search for differences between clump and RGB stars at the same $\Delta v$ (i.e., same mean stellar density). To estimate the influence of the stellar mass on $\Gamma_{0}\left(v_{\max }\right)$, we determine the mass from scaling relations for the global oscillation parameters $\left(\Delta v\right.$ and $\left.v_{\max }\right)$ and the effective temperature (taken from the APOKASC measurements, see Pinsonneault et al. 2014).

The dependence of $\Gamma_{0}\left(v_{\max }\right)$ on the large separation is shown in Fig. 3, where we find very similar results for both methods. The main difference is a larger dispersion for the FREQ method, which is due to larger uncertainties. We also find a small but clear mass dependence, regardless of the stars evolutionary status. This is particularly obvious for helium-burning stars. The values of $\Gamma_{0}\left(v_{\max }\right)$ for clump stars show indeed a clear dependence on $\Delta v$, especially in the secondary clump, which is a consequence of the mass dependence. At fixed $\Delta v$, a star with a higher mass has a higher $\Gamma_{0}\left(v_{\max }\right)$ value. The influence of the stellar mass on the pressure mode widths was not widely considered by previous theoretical studies. It is therefore difficult to assess the implications of such a behavior. This result suggests that more theoretical work is needed to understand the contributions of the different physical mechanisms behind mode damping.

We also note that, for stars with similar masses and the same large separation, the global radial mode widths in the clump are significantly larger than those on the RGB as was previously mentioned by Corsaro et al. (2012). Given the clear difference in behavior between the two evolutionary states, we separate them in the analysis that follows.

Finally, two minor features can be observed. Firstly, low $\Gamma_{0}\left(v_{\max }\right)$ values are observed for evolved RGB stars (bottom part of Fig. 3), which can be explained by their low $T_{\text {eff }}$ (see Sect. 4.2). The second one concerns the way $\Gamma_{0}\left(v_{\max }\right)$ evolves along the RGB: both methods show a non-monotonous variation of this parameter with $\Delta v$. It certainly indicates that the large separation is not a good proxy of the stellar evolution for studying the mode width. Further studies are needed in order to understand this particular behavior.

\subsection{The $\Gamma_{0}\left(v_{\max }\right)-T_{\text {eff }}$ relation}

According to theoretical predictions, the stellar linewidths should strongly depend on the stellar effective temperature (e.g., Chaplin et al. 2009; Belkacem et al. 2012). Such a strong correlation has already been confirmed for main sequence and subgiant solar-like pulsators (Baudin et al. 2011; Appourchaux et al. 2012). For red giants, however, the radial mode widths seem to be less strongly correlated with $T_{\text {eff }}$ (Baudin et al. 2011; Corsaro et al. 2012, 2015) or even independent of it (Handberg et al. 2017). As an example, to take mode widths measurements from both main sequence and red giant stars into account, Corsaro et al. (2012) had to introduce an exponential dependence of the mode widths with $T_{\text {eff }}$. Here, we analyze the $\Gamma_{0}\left(v_{\max }\right)-T_{\text {eff }}$ correlation in order to confirm 

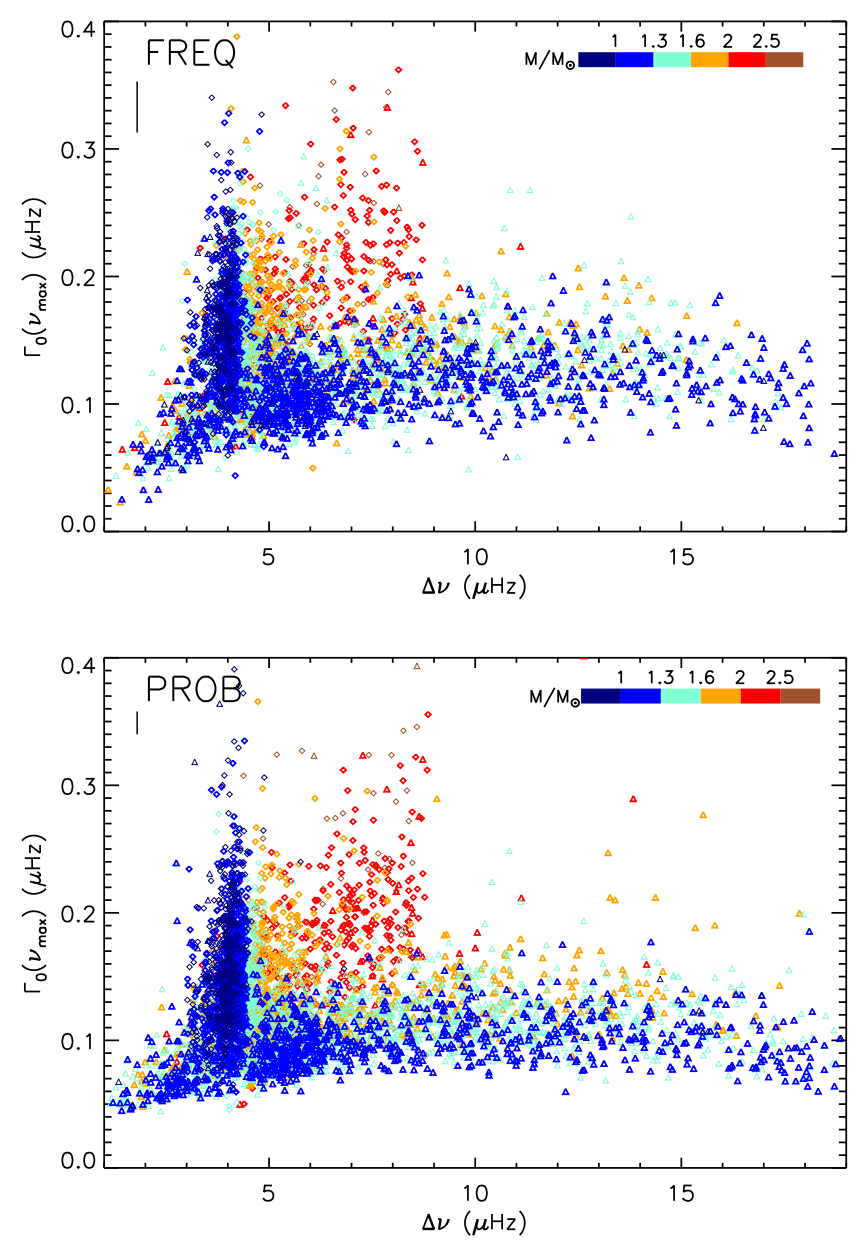

Fig. 3. Global radial mode width $\left(\Gamma_{0}\left(v_{\max }\right)\right)$ as a function of the large separation $(\Delta v)$ for the FREQ method (top) and for the PROB method (bottom), with the stellar mass color-coded. The diamond and triangle symbols indicate clump and RGB stars, respectively. The mean uncertainty in $\Gamma_{0}\left(v_{\max }\right)$ is given by the vertical black line in the upper left corner.

or revoke the previous results. For this, we used the effective temperatures listed in the latest APOKASC catalog (see e.g., Pinsonneault et al. 2014).

The results are shown separately for RGB and clump stars in Fig. 4; their values are consistent with the ones from previous studies (Baudin et al. 2011; Corsaro et al. 2012, 2015). On the RGB, we note a double dependence: firstly, the radial mode width decreases with decreasing $T_{\text {eff }}$; secondly, at a given $T_{\text {eff }}$, the radial mode width increases with increasing mass. This shows that both the stellar mass and effective temperature have an influence on $\Gamma_{0}\left(v_{\max }\right)$, which was not reported in previous analyses (e.g., Baudin et al. 2011).

In order to establish the degree of the dependence, we fit the linewidths as a function of $T_{\text {eff }}$. On the RGB, we find $\Gamma_{0}\left(v_{\max }\right)$ to vary with $T_{\text {eff }}{ }^{5.28 \pm 0.17}$ for the mode widths obtained with the FREQ method and $T_{\text {eff }}{ }^{4.74 \pm 0.13}$ for the ones obtained with the PROB method. In order to study the impact of the mass dependence on these fits, we perform several fits for stars with similar masses. The results can be seen on Table 2. They are consistent with each other.

The global mass dependence on the RGB can also be estimated with the same procedure. We find $\Gamma_{0}\left(v_{\max }\right)$ to scale with $M^{0.14 \pm 0.13}$ for the mode widths obtained with the FREQ method and with $M^{0.27 \pm 0.13}$ for the ones obtained with the PROB method.
Here also, the temperature can have an influence on these measured dependencies. We then perform several fits for stars with similar temperature values to measure this impact. The results can be seen in Table 3. The mass dependence is identified as being more important for stars with high $T_{\text {eff }}$ compared to stars with low $T_{\text {eff }}$. Both methods show the same behavior, the only difference is in the magnitude of the dependence that is higher for the PROB values at high $T_{\text {eff }}$. We also notice an important variation of the dependence as a function of the range of $T_{\text {eff }}$ selected. This phenomenon indicates a low reliability of the global mass dependence previously measured as shown by their large uncertainties. However, the variation of the results can also be caused by the low number of stars with high mass values present at low $T_{\text {eff }}$ leading to less accurate fits. These results show that a precise characterization of the mass dependence independent of the influence of the temperature is currently difficult for RGB stars and that the performed measurements have to be considered as an approximation. This problem could be overcome by the use of population synthesis in order to identify and correct all possible biases but this is beyond the scope of this paper.

For clump stars, we find $\Gamma_{0}\left(v_{\max }\right)$ to vary with $T_{\mathrm{eff}}^{4.10 \pm 0.17}$ for the FREQ method and $T_{\text {eff }}{ }^{3.82 \pm 0.21}$ for the PROB method, which correspond to lower exponents than for RGB stars. This can be explained by the fact that clump stars with similar masses have similar effective temperatures, as can be seen in Fig. 4. The presence of high-mass stars with high $T_{\text {eff }}$ will then artificially modify the measured dependence in that specific case. In fact, the absence of important $T_{\text {eff }}$ evolution during the clump phase on the contrary to the RGB does not readily allow us to disentangle the mass and temperature dependencies.

The exponent of the observed global power laws turns out to be much smaller for red giants than for less-evolved stars, with exponents between 15.3 \pm 1.9 (Lund et al. 2017) and $16.7 \pm 1.8$ (Appourchaux et al. 2012). Moreover, the observed relationship seems to differ from theoretical expectations. The small exponent we find for the power law does not appear to correspond to the high value predicted by theory (10.8 following Belkacem et al. 2012). However, previous theoretical work, such as the one performed by Belkacem et al. (2012), took main sequence stars as well as red giants into account to deduce the $\Gamma_{0}\left(v_{\max }\right)-T_{\text {eff }}$ relation. It is therefore not relevant to compare the theory to the exponent we measured based on red giants only.

Although our sample covers a limited range in effective temperature, so that any possible bias in $T_{\text {eff }}$ may affect the fit, our results are based on the largest sample of red giants analyzed so far when addressing radial mode widths and, consequently, we may consider them to benefit from a high statistical reliability.

Finally, we investigate the impact of the stellar metallicity on the mode width but find no significant influence (see Appendix B).

\subsection{Individual mode width variation with frequency}

The variation of the individual mode widths $\left(\Gamma_{0}\right)$ with frequency for a single star was previously analyzed for subgiants and main sequence solar-type pulsators (Appourchaux et al. 2014; Lund et al. 2017). This variation exhibits a similar behavior for all solar-type pulsators with low effective temperature. It is characterized by a global increase of the mode width with increasing frequency along with a small $\Gamma_{0}$ dip around $v_{\max }$ (e.g., Lund et al. 2017). The origin of the dip was theoretically investigated by Belkacem et al. (2012) who attributed it to the competition 

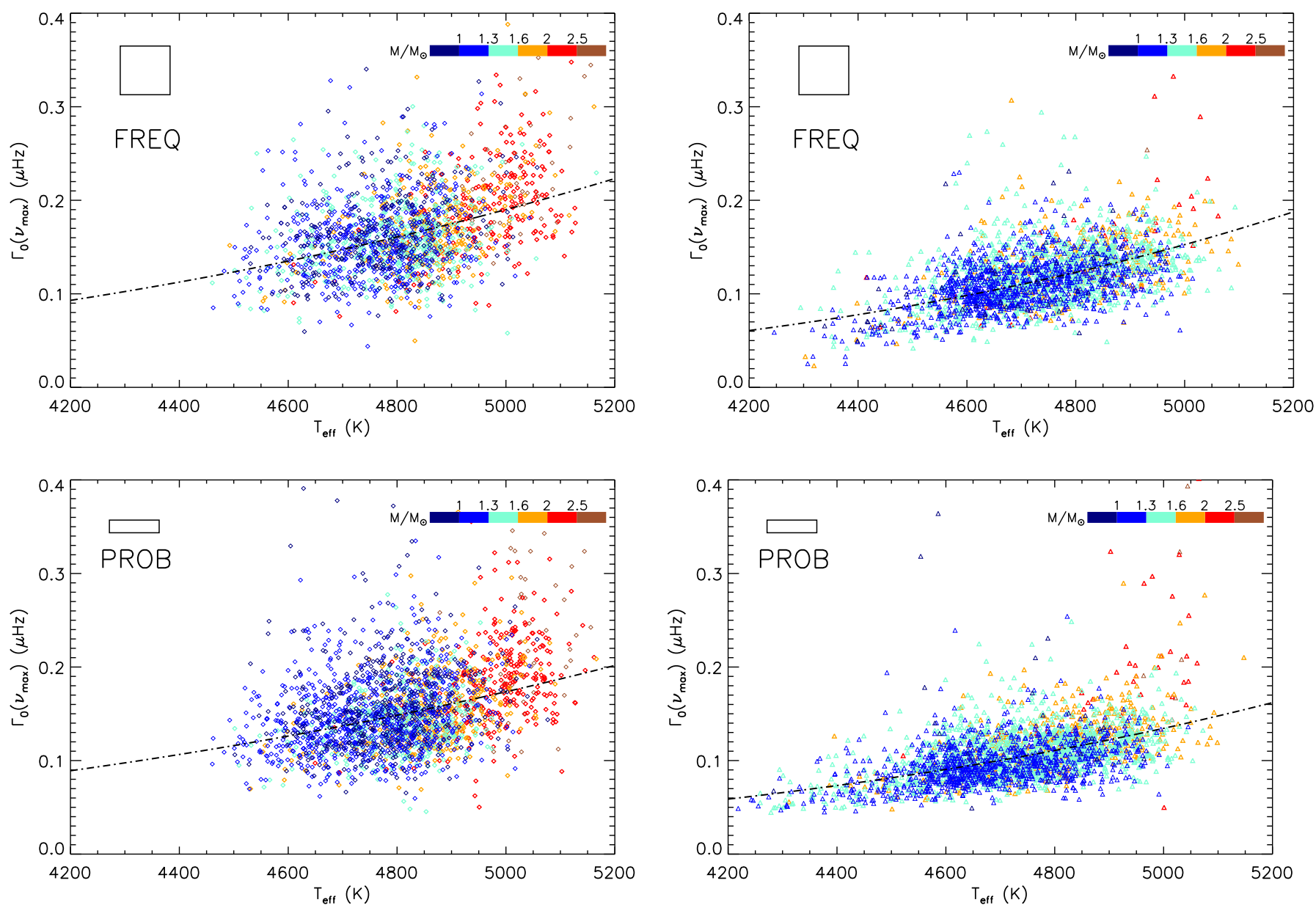

Fig. 4. Global radial mode widths $\Gamma_{0}\left(v_{\max }\right)$ as a function of the effective temperatures $T_{\text {eff }}$ for the FREQ method (top) and for the PROB method (bottom). Clump and RGB stars are shown in the left and right panels, respectively, with their mass color-coded. The black squares indicate the typical uncertainties and the black dashed-dotted lines give the width-temperature relation fitted for each set of stars.

Table 2. Temperature dependence of $\Gamma_{0}\left(v_{\max }\right)$ on the RGB for stars with different mass values.

\begin{tabular}{lcccc}
\hline \hline$M / M_{\odot}$ & $1.0-1.2$ & $1.2-1.4$ & $1.4-1.6$ & $1.6-1.8$ \\
\hline$\alpha_{T}$ (FREQ) & $5.71 \pm 0.39$ & $5.03 \pm 0.45$ & $5.41 \pm 0.59$ & $5.78 \pm 0.69$ \\
$\alpha_{T}$ (PROB) & $4.55 \pm 0.27$ & $5.29 \pm 0.38$ & $4.54 \pm 0.40$ & $4.65 \pm 0.54$ \\
\hline
\end{tabular}

Notes. The temperature dependence of $\Gamma_{0}\left(v_{\max }\right)$ is modeled as $\Gamma_{0}\left(v_{\max }\right) \propto T_{\text {eff }^{\alpha}}^{\alpha_{T}}$.

between the perturbation of the turbulent pressure and the perturbation of entropy. These two physical mechanisms have opposite impacts: one contributes to the damping and the other to the driving, so that they compensate each other. The maximum compensation between the two terms happens around $v_{\max }$, where it induces a dip in the global increase of the mode width. Following that study, the $\Gamma_{0}$ dip should be observed for the mode widths in all solar-like pulsators, including red giant stars. This was already pointed out by Corsaro et al. (2015) for most of the 19 low-RGB stars they analyzed. The purpose of this section is to see whether this behavior can be observed in a larger sample.

We estimate the global shape of the $\Gamma_{0}$ variation with frequency by using a collapsogram on the mode radial order. This corresponds to the calculation of the mean ratio between $\Gamma_{0}$ and $\Gamma_{0}\left(v_{\max }\right)$ as a function of the radial mode order centered on $v_{\max }$.
Table 3. Mass dependence of $\Gamma_{0}\left(v_{\max }\right)$ on the RGB for stars with different effective temperature values.

\begin{tabular}{cccc}
\hline \hline$T_{\text {eff }}(\mathrm{K})$ & $4500-4700$ & $4700-4900$ & $4900-5100$ \\
\hline$\alpha_{M}$ (FREQ) & $0.112 \pm 0.051$ & $0.138 \pm 0.036$ & $0.502 \pm 0.069$ \\
$\alpha_{M}$ (PROB) & $0.066 \pm 0.048$ & $0.251 \pm 0.034$ & $0.906 \pm 0.063$ \\
\hline
\end{tabular}

Notes. Mass dependence of $\Gamma_{0}\left(v_{\max }\right)$ on the RGB modeled as $\Gamma_{0}\left(v_{\max }\right) \propto$ $\left(M / M_{\odot}\right)^{\alpha_{M}}$.

Firstly, we identify the radial order $n$ of each radial mode and the value of $n$ for the radial mode closest to the maximum oscillation power. This order is called $n_{\max }$ and is obtained with the following relation $n_{\max }=\Delta v / v_{\max }-\varepsilon$, where $\varepsilon$ is the offset of the asymptotic pattern. Secondly, we calculate the ratio between the individual mode widths and $\Gamma_{0}\left(v_{\max }\right)$. Finally, we compute collapsograms by taking the mean value of these ratios with respect to the relative radial order $n-n_{\max }$. This was done for clump and RGB stars separately, following the differences that arise between the two evolutionary states. The results are shown in Fig. 5.

Both methods show an increase of the relative radial mode width with a slow-down around $n_{\max }$ for RGB stars only, which could correspond to the $\Gamma_{0}$ dip observed for main sequence and subgiant solar-like pulsators even if the large uncertainties do not allow firm conclusions. For the FREQ method, we also note 

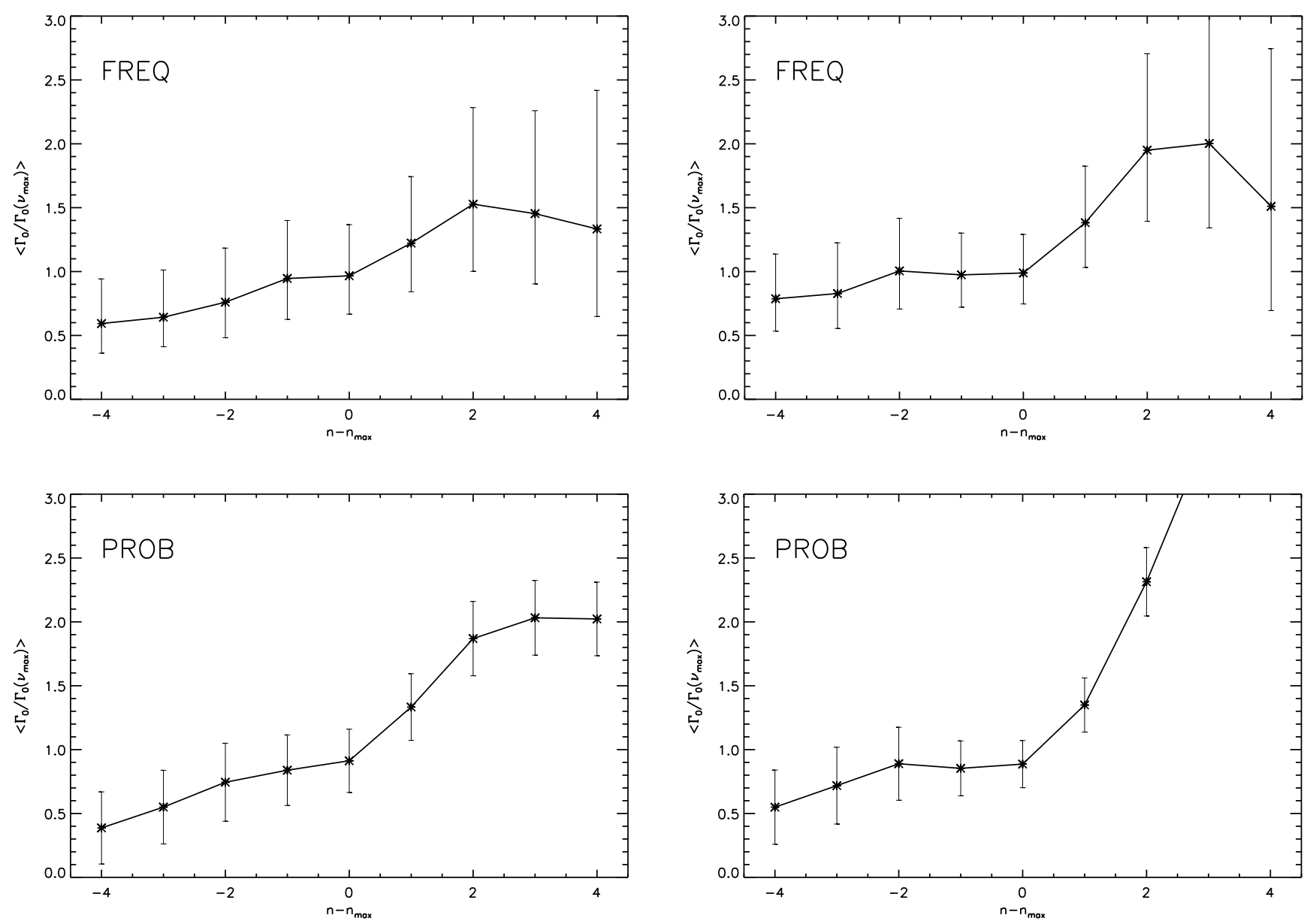

Fig. 5. Collapsogram of the relative radial mode width as a function of the radial mode order $n-n_{\max }$ for the FREQ method (top) and for the PROB method (bottom). Clump and RGB stars are shown in the left and right panels, respectively. The error bars correspond to the $1 \sigma$ uncertainties.

an apparent stabilization or even a decrease of the relative radial mode width at high radial orders (above $n_{\max }+2$ ), which is not found in other solar-like pulsators. However, the large uncertainties for the higher radial orders and the low number of stars for which we obtain a radial mode width measurement at these radial orders question the physical meaningfulness of this observation. In fact, this behavior is not observed for the mode width obtained with the PROB method. Instead, we note a continuous increase of the mode width, casting doubts on the reality of the behavior of the relative radial mode width at high radial order for the FREQ method.

As mentioned before, the increase in the relative radial mode width undergoes a slow-down near $n_{\max }$ for RGB stars in both methods. However, this behavior does not appear for the clump stars in the FREQ method and is significantly reduced in the PROB method. This could indicate that the dominant physical mechanism behind mode damping changes between the RGB and clump evolutionary states. However, the large uncertainties prevent us from drawing a firm conclusion and further studies are needed in order to confirm this behavior.

Finally, we check the influence of the stellar mass, surface gravity and $T_{\text {eff }}$ on the result. We find no significant differences when restricting the collapsogram to either low-mass stars or high-mass stars. Therefore, we conclude that the mass influence on these results is negligible. When restricting the collapsograms to low- $T_{\text {eff }}$ stars or stars with low surface gravity, the dip appears more distinctly but, considering the error bars, no firm conclusions could be drawn.

\section{Mode amplitudes}

In this section, we discuss the results we obtain for the relative amplitudes and compare them with theoretical predictions and previous observational results.

\subsection{Amplitude variation with stellar evolution}

The variation of $A_{0, \text { bol }}$ with $v_{\max }$ is shown in Fig. 6 for both methods. As for the mode widths, the maximum mode amplitudes obtained with the two methods show a similar behavior. The decrease of the stellar envelope density with stellar luminosity is manifested by the increase of $A_{0, \text { bol }}$ with decreasing $v_{\text {max }}$. This decrease in envelope density enables a more vigorous convection and, consequently, a more efficient mode excitation. We also note, for both samples and both evolutionary states, a clear mass dependence: stars with a higher mass have a lower maximum mode amplitude, as previously noticed (Huber et al. 2010, 2011; Mosser et al. 2011a, 2012a; Stello et al. 2011; Kallinger et al. 2014). However, we can clearly see that the mass dependence is present in an equivalent manner for both evolutionary states.

Contrary to the global behavior, the measured amplitude values differ between the two methods. For RGB stars with low $v_{\max }$, the FREQ amplitudes are globally underestimated by about $20 \%$ with respect to the PROB values. On the other hand, clump stars are less affected, leading to a small overestimation of the amplitudes we obtained with the FREQ method compared to the 

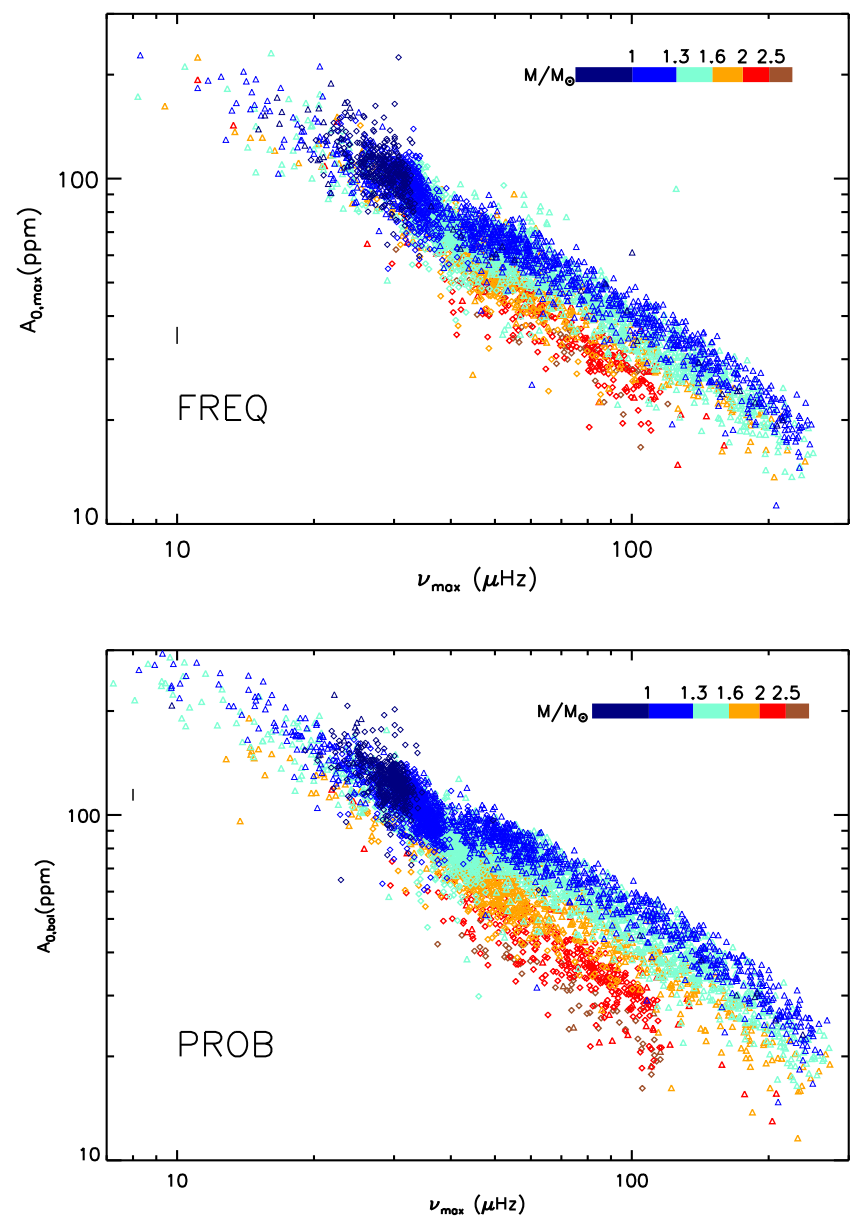

Fig. 6. Bolometric amplitudes $A_{0, \mathrm{bol}}$ as a function of $v_{\max }$ for the FREQ method (top) and for the PROB method (bottom). The color code indicates stellar mass. The diamond and triangle symbols indicate clump and RGB stars, respectively. The black line corresponds to the mean uncertainties.

PROB method for stars with high $v_{\max }$. The origin of this difference is not known.

One last thing to observe is the similarity between clump and RGB star amplitudes. At similar mass and seismic parameters, stars in both evolutionary states have approximately the same maximum amplitude (Fig. 6).

\subsection{The amplitude $-L / M$ relation}

Following Kjeldsen \& Bedding (1995, 2011), Houdek et al. (1999) and later Samadi et al. (2012), the bolometric amplitude of solar-like oscillations is expected to scale as

$A_{0, \mathrm{bol}} \propto \tau_{\max }^{1 / 2}\left(\frac{L}{M}\right)^{a} \Delta v^{b}$

In order to test this theoretical scaling we need an estimate for $L$, which is provided by the scaling relations of $v_{\max } \propto M / R^{2} / \sqrt{T_{\text {eff }}}$ (e.g., Brown 1991; Kjeldsen \& Bedding 1995) and $L \propto R^{2} T_{\text {eff }}{ }^{4}$. It follows (e.g., Baudin et al. 2011),

$L \propto M T_{\mathrm{eff}}^{7 / 2} v_{\max }^{-1}$

Since $\Delta v$ is expected to scale as $\Delta v \propto \sqrt{M / R^{3}}$ (e.g., Ulrich 1986; Brown \& Gilliland 1994) we can substitute $\Delta v$ in Eq. (7). Assuming $\Gamma_{0}\left(v_{\max }\right) \propto M^{c} T_{\text {eff }}^{d}$ we can then write,
$A_{0, \mathrm{bol}} \propto L^{a-3 b / 4} M^{-a+b / 2-c / 2} T_{\mathrm{eff}}^{-d / 2+3 b}$

From this development, it can clearly be seen that the $b$ exponent, which represents the $\Delta v$ dependence (Eq. (7)), does not produce an important influence in the departure from the $L / M$ power law. On the contrary, the influence of the $c$ exponent, introduced by $\Gamma$, amplifies the departure from a power law in $L / M$.

In order to simplify the approach, we consider that the relative variation of the effective temperature on the RGB and in the clump is so small compared to the variation in luminosity that we can neglect the $T_{\text {eff }}$ dependence. Therefore, we performed a fit of $A_{0, \text { bol }}$ as power law of $L$ and $M$ only. We find a variation in $L^{0.77 \pm 0.06} M^{-1.25 \pm 0.14}$ with the FREQ method, and in $L^{0.70 \pm 0.06} M^{-1.40 \pm 0.13}$ with the PROB method. In order to evaluate the validity of the previous hypothesis leading to neglect the $T_{\text {eff }}$ influence, the variation of the mode amplitude with $L, M$ and $T_{\text {eff }}$ was also measured. We find a variation in $L^{0.70 \pm 0.07} M^{-1.03 \pm 0.15} T_{\text {eff }}{ }^{-4.79 \pm 1.36}$ with the FREQ method, and in $L^{0.61 \pm 0.06} M^{-1.14 \pm 0.14} T_{\text {eff }}{ }^{-5.48 \pm 1.18}$ with the PROB method. The dependence in $L$ and $M$ are less important, as expected, but consistent with the ones we previously found, considering the error bars. In contrast to these results, we can see that the measured $T_{\text {eff }}$ dependence is very important. However, the large error bars on the exponent and the lack of important impact on the other dependencies lead us to confirm the simplification hypothesis and neglect the $T_{\text {eff }}$ dependence.

To assess the impact of the clump stars on the fits, we also consider the RGB stars alone and find significant differences: $A_{0, \text { bol }}$ varying as $L^{0.84 \pm 0.08} M^{-1.34 \pm 0.27}$ with the FREQ method, and in $L^{0.82 \pm 0.07} M^{-1.61 \pm 0.27}$ with the PROB method. As stated above, seismic and stellar parameters of stars do not evolve much during the helium burning phase, on the contrary to the RGB phase. It follows that clump stars with similar masses will have simi$\operatorname{lar} A_{0, \mathrm{bol}}$ values, thus making it difficult to disentangle the luminosity and mass dependence for these stars. The fit performed only on RGB stars allows us to get rid of a possible influence of one dependence on the other, and is therefore the one considered throughout the rest of the article.

The exponents we find for the luminosity are slightly larger than the ones theoretically predicted (Samadi et al. 2012), but agree with previous measurements (Mosser et al. 2010, 2012a; Huber et al. 2011; Stello et al. 2011; Corsaro et al. 2013; Kallinger et al. 2014). A possible explanation for the difference between the predicted and observed exponents is the strong influence of non-adiabatic effects on the mode driving in red giants. Indeed, the theoretical predictions previously mentioned do not rely on fully non-adiabatic models. This type of modeling has not yet been performed (Samadi et al. 2013).

The observed exponents in our fits help to constrain the theoretical predictions. We derive the exponents $a=2.13 \pm 1.16$ and $b=1.72 \pm 1.66$ for the FREQ method and $a=2.77 \pm 1.14$ and $b=2.60 \pm 1.62$ for the PROB method. We note that the relationship between the mode width $\Gamma_{0}\left(v_{\max }\right)$ and the stellar mass helps us to understand the different exponents in $L$ and $M^{-1}$. However, these exponents are significantly larger than the ones predicted by theoretical works, corresponding to $a=1.55 \pm 0.09$ and $b=0.50 \pm 0.03$ (Samadi et al. 2012). Even if large uncertainties are present in our results, the discrepancies between observations and theoretical works show that more studies are needed in order to produce realistic models describing the physical mechanisms, which govern mode excitation. 

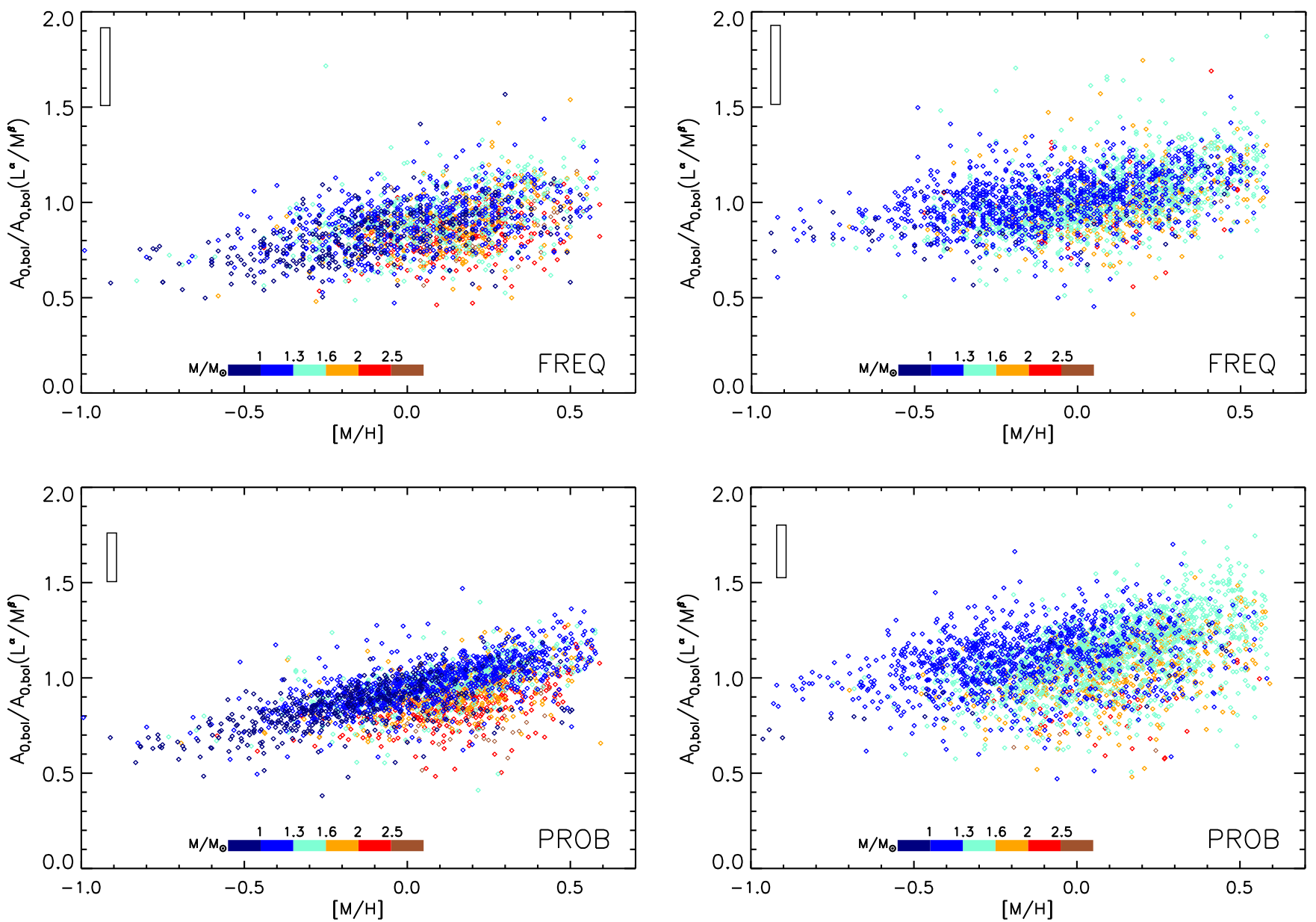

Fig. 7. Bolometric amplitudes $\left(A_{0, \mathrm{bol}}\right)$ corrected for the $L$ and $M$ dependencies as a function of the stellar metallicity for the FREQ method (top) and the PROB method (bottom), with the stellar mass color-coded. Clump stars are shown in the left panels and RGB stars in the right panels. The black rectangle corresponds to the typical uncertainties.

\subsection{Evidence of a metallicity influence}

Several theoretical studies suggest that stellar metallicity has an influence on the mode amplitude (Houdek et al. 1999; Samadi et al. 2010b). In order to test this, we plot the mode amplitudes, cleared of the other dependencies we characterized previously, as a function of the stellar metallicity (Fig. 7). The metallicities are taken from the APOKASC catalog, providing $[\mathrm{M} / \mathrm{H}]$ measurements for 4367 objects from which an $A_{0, \text { bol }}$ and $\Gamma_{0}\left(v_{\max }\right)$ value could be measured with the FREQ method and for 5339 objects from the PROB method.

The identification of a metallicity dependence requires the suppression of the previously identified luminosity and mass dependencies. We therefore use the fits from Sect.5.2 to correct the amplitudes for the influence of the previously characterized $L$ and $M$. We estimate the dependence of the normalized amplitudes on metallicity separately for clump and RGB stars in order to remove a potential influence from the stellar evolutionary status. We find a clear increase of the mode amplitudes with increasing metallicity for both evolutionary states and both methods in agreement with the previous analysis (Mosser et al. 2012a). However, we find a slightly higher dependence in our data: an increase of one dex in metallicity causes an increase of more than $20 \%$ in amplitude. More precisely, for the FREQ method, the 1 dex increase corresponds to a $25 \% \pm 3 \%$ increase in amplitude for RGB stars and $27 \% \pm 3 \%$ for clump stars. For the PROB method, the rise corresponds to $45 \% \pm 2 \%$ for RGB stars and $49 \% \pm 2 \%$ for clump stars, which is slightly higher than the results we obtain with the other method.

The overall results are in agreement with theoretical predictions (e.g., Houdek et al. 1999; Samadi et al. 2010a,b), which suggest larger pressure mode amplitudes for metal-rich stars than for metal-poor stars. Even though we still have to investigate if our observational results are comparable to these predictions in a more quantitative way, such an agreement indicates that the global understanding of the variation of the mode amplitude with the stellar physical parameters is already quite good.

These results are also in agreement with the metallicity influence observed for the granulation, which correspond to an increase of the granulation amplitude with the stellar metallicity (e.g., Corsaro et al. 2017), since mode and granulation amplitude are linked (Kjeldsen \& Bedding 2011). However, it appears that the metallicity influence is much weaker for the mode amplitude compared to the one found by Corsaro et al. (2017) for granulation amplitude.

\section{Conclusion}

In this study, we measure the radial mode widths and amplitudes in thousands of red giant stars. Our results emphasize a relationship between the radial mode width and the effective temperature, which can be modeled by a power law. The exponent of the power law is, however, much smaller than those previously observed for subgiants and main sequence stars but is 
consistent with previous measurements done with red giants. We also find a significant mass dependence of the radial mode width and that the mode widths are sensitive to the evolutionary state of a star, with clump stars generally having broader radial modes than RGB stars, as previously shown by Corsaro et al. (2012). The mode widths are directly related to mode damping meaning that their measurements offer new constraints on this parameter. Since our results are not completely compatible with theoretical predictions, they suggest that the contributions of the different physical mechanisms to mode damping in red giants have to be specified and work in a manner that is different from that in main sequence stars. Further theoretical studies will be necessary to fully exploit the new constraints we find for this parameter.

On the contrary, our results for the mode amplitudes confirm and extend previous ones, in particular on the influence of the mass and the relation between the mode amplitudes and the luminosity-to-mass ratio. We can also confirm a clear influence of the stellar metallicity on the mode amplitudes: an increase in metallicity causes an increase of the mode amplitude. The theoretical prediction of such a behavior seems to indicate that the physical mechanism behind mode excitation is already well understood. However, differences between the observed dependence (especially the dependence with the stellar mass and luminosity) and theoretical predictions show that some further studies are necessary in order to develop realistic models taking all non-adiabatic effects into account.

Acknowledgements. MV acknowledges funding by the Portuguese Science and Technology Foundation (FCT) through the grant with reference CIAAUP-03/2016-BPD, in the context of the project FIS/04434, co-funded by FEDER through the program COMPETE. MSC was supported by FCT through national funds (Investigador contract of reference IF/00894/2012 and UID/FIS/04434/2013) and by FEDER through COMPETE2020 (POCI-010145-FEDER-030389 and POCI-01-0145- FEDER-007672).

\section{References}

Appourchaux, T. 2014, in A Crash Course on Data Analysis in Asteroseismology, eds. P. L. Pallé, \& C. Esteban (Cambridge, UK: Cambridge University Press), 123

Appourchaux, T., Benomar, O., Gruberbauer, M., et al. 2012, A\&A, 537, A134 Appourchaux, T., Antia, H. M., Benomar, O., et al. 2014, A\&A, 566, A20 Baglin, A., Auvergne, M., Barge, P., et al. 2006, ESA SP, 1306, 33 Ballot, J., Barban, C., \& van't Veer-Menneret, C. 2011, A\&A, 531, A124 Balmforth, N. J. 1992, MNRAS, 255, 639

Barban, C., Baudin, F., Mosser, B., et al. 2010, Astron. Nachr., 331, 1016

Baudin, F., Barban, C., Belkacem, K., et al. 2011, A\&A, 529, A84

Beck, P. G., Bedding, T. R., Mosser, B., et al. 2011, Science, 332, 205

Bedding, T. R., Huber, D., Stello, D., et al. 2010, ApJ, 713, L176

Bedding, T. R., Mosser, B., Huber, D., et al. 2011, Nature, 471, 608

Belkacem, K., Samadi, R., Goupil, M. J., Kupka, F., \& Baudin, F. 2006, A\&A, 460, 183

Belkacem, K., Dupret, M. A., Baudin, F., et al. 2012, A\&A, 540, L7
Borucki, W. J., Koch, D., Basri, G., et al. 2010, Science, 327, 977

Brown, T. M. 1991, in ASP Conf. Ser., 20, 139

Brown, T. M., \& Gilliland, R. L. 1994, ARA\&A, 32, 37

Chaplin, W. J., Houdek, G., Karoff, C., Elsworth, Y., \& New, R. 2009, A\&A, 500, L21

Corsaro, E., Stello, D., Huber, D., et al. 2012, ApJ, 757, 190

Corsaro, E., Fröhlich, H.-E., Bonanno, A., et al. 2013, MNRAS, 430, 2313

Corsaro, E., De Ridder, J., \& García, R. A. 2015, A\&A, 579, A83

Corsaro, E., Mathur, S., García, R. A., et al. 2017, A\&A, 605, A3

De Ridder, J., Barban, C., Baudin, F., et al. 2009, Nature, 459, 398

Dupret, M. A., Barban, C., Goupil, M.-J., et al. 2006, ESA SP, 624, 97

Dupret, M., Belkacem, K., Samadi, R., et al. 2009, A\&A, 506, 57

Elsworth, Y., Hekker, S., Basu, S., \& Davies, G. R. 2017, MNRAS, 466, 3344

Feroz, F., Hobson, M. P., \& Bridges, M. 2009, MNRAS, 398, 1601

Goldreich, P., \& Kumar, P. 1991, ApJ, 374, 366

Handberg, R., Brogaard, K., Miglio, A., et al. 2017, MNRAS, 472, 979

Hon, M., Stello, D., \& Yu, J. 2017, MNRAS, 469, 4578

Houdek, G. 2012, ASP Conf. Ser., 462, 7

Houdek, G., Balmforth, N. J., Christensen-Dalsgaard, J., \& Gough, D. O. 1999, A\&A, 351, 582

Houdek, G., Trampedach, R., Aarslev, M. J., \& Christensen-Dalsgaard, J. 2017, MNRAS, 464, L124

Huber, D., Bedding, T. R., Stello, D., et al. 2010, ApJ, 723, 1607

Huber, D., Bedding, T. R., Stello, D., et al. 2011, ApJ, 743, 143

Jeffreys, H. 1998, in Theory of Probability, Oxford Classic Texts in the Physical Sciences (Oxford: Clarendon Press)

Kallinger, T., Guenther, D. B., Matthews, J. M., et al. 2008, A\&A, 478, 497

Kallinger, T., Mosser, B., Hekker, S., et al. 2010a, A\&A, 522, A1

Kallinger, T., Weiss, W. W., Barban, C., et al. 2010b, A\&A, 509, A77

Kallinger, T., Hekker, S., Mosser, B., et al. 2012, A\&A, 541, A51

Kallinger, T., De Ridder, J., Hekker, S., et al. 2014, A\&A, 570, A41

Kjeldsen, H., \& Bedding, T. R. 1995, A\&A, 293, 87

Kjeldsen, H., \& Bedding, T. R. 2011, A\&A, 529, L8

Lund, M. N., Silva Aguirre, V., Davies, G. R., et al. 2017, ApJ, 835, 172

Mosser, B., \& Appourchaux, T. 2009, A\&A, 508, 877

Mosser, B., Belkacem, K., Goupil, M., et al. 2010, A\&A, 517, A22

Mosser, B., Barban, C., Montalbán, J., et al. 2011a, A\&A, 532, A86

Mosser, B., Belkacem, K., Goupil, M., et al. 2011b, A\&A, 525, L9

Mosser, B., Elsworth, Y., Hekker, S., et al. 2012a, A\&A, 537, A30

Mosser, B., Goupil, M. J., Belkacem, K., et al. 2012b, A\&A, 540, A143

Mosser, B., Benomar, O., Belkacem, K., et al. 2014, A\&A, 572, L5

Mosser, B., Pinçon, C., Belkacem, K., Takata, M., \& Vrard, M. 2017, A\&A, 600, A1

Pinsonneault, M. H., Elsworth, Y., Epstein, C., et al. 2014, ApJS, 215, 19

Samadi, R., Georgobiani, D., Trampedach, R., et al. 2007, A\&A, 463, 297

Samadi, R., Ludwig, H.-G., Belkacem, K., et al. 2010a, A\&A, 509, A16

Samadi, R., Ludwig, H.-G., Belkacem, K., Goupil, M. J., \& Dupret, M.-A. 2010b, A\&A, 509, A15

Samadi, R., Belkacem, K., Dupret, M.-A., et al. 2012, A\&A, 543, A120

Samadi, R., Belkacem, K., Dupret, M.-A., et al. 2013, EPJ Web Conf., 43, 03008

Samadi, R., Belkacem, K., \& Sonoi, T. 2015, EAS Pub. Ser., 73, 111

Stello, D., Huber, D., Kallinger, T., et al. 2011, ApJ, 737, L10

Stello, D., Huber, D., Bedding, T. R., et al. 2013, ApJ, 765, L41

Toutain, T., \& Appourchaux, T. 1994, A\&A, 289, 649

Ulrich, R. K. 1986, ApJ, 306, L37

Vrard, M., Mosser, B., Barban, C., et al. 2015, ApJ, 579, A84

Vrard, M., Mosser, B., \& Samadi, R. 2016, A\&A, 588, A87

Walker, G., Matthews, J., Kuschnig, R., et al. 2003, PASP, 115, 1023 


\section{Appendix A: Precise mode-fitting results for each individual star}

The FREQ method allows us to realize precise mode fitting of several radial modes in the oscillation spectra of each of the
5221 stars that were analyzed in this study. The results are available in the form of several files (one for each star) at the CDS. An example of the shape of the different files is given in Table A.1 for the star KIC 1027337.

Table A.1. Radial mode parameters for the star KIC 1027337.

\begin{tabular}{cccccccc}
\hline \hline $\begin{array}{c}\text { Frequency } \\
(\mu \mathrm{Hz})\end{array}$ & $\begin{array}{c}\delta \text { Frequency } \\
(\mu \mathrm{Hz})\end{array}$ & $\begin{array}{c}\Gamma \\
(\mu \mathrm{Hz})\end{array}$ & $\begin{array}{c}\delta_{U} \Gamma \\
(\mu \mathrm{Hz})\end{array}$ & $\begin{array}{c}\delta_{L} \Gamma \\
(\mu \mathrm{Hz})\end{array}$ & $\begin{array}{c}\text { Amplitude } \\
(\mathrm{ppm})\end{array}$ & $\begin{array}{c}\delta_{U} \text { Amplitude } \\
(\mathrm{ppm})\end{array}$ & $\begin{array}{c}\delta_{L} \text { Amplitude } \\
(\mathrm{ppm})\end{array}$ \\
\hline 56.94 & 0.0136 & 0.0815 & 0.0413 & 0.0274 & 25.76 & 14.079 & 9.090 \\
63.51 & 0.0091 & 0.0702 & 0.0190 & 0.0149 & 34.37 & 10.625 & 8.080 \\
70.47 & 0.0086 & 0.1463 & 0.0186 & 0.0165 & 53.81 & 7.200 & 6.348 \\
77.39 & 0.0105 & 0.1280 & 0.0342 & 0.0270 & 68.25 & 21.831 & 16.461 \\
84.36 & 0.0142 & 0.1371 & 0.0367 & 0.0290 & 40.13 & 12.597 & 9.557 \\
91.65 & 0.0305 & 0.1649 & 0.0957 & 0.0606 & 18.46 & 10.701 & 6.777 \\
\hline
\end{tabular}

Notes. Tables for the 5221 stars that were used in this study are available at the CDS. The columns correspond to, from left to right, the mode frequencies, the $1 \sigma$ uncertainties on this parameter, the mode widths $(\Gamma)$, the upper $1 \sigma$ uncertainties on this parameter, its lower $1 \sigma$ uncertainties, the mode amplitudes, the upper $1 \sigma$ uncertainties on this parameter and its lower $1 \sigma$ uncertainties.

\section{Appendix B: Metallicity influence on mode width values}

The influence of the metallicity on the mode width was previously mentioned as a possibility (Baudin, priv. comm.); we subsequently investigated it in this study. Figure B.1 shows the variation of $\Gamma_{0}\left(v_{\max }\right)$ as a function of the large separation for the PROB method and with a color code indicating the star metallicities. The metallicities are taken from the APOKASC catalog, providing $[\mathrm{M} / \mathrm{H}]$ measurements for 4367 objects from which a $A_{0, \text { bol }}$ and $\Gamma_{0}\left(v_{\max }\right)$ value could be measured with the FREQ method and for 5339 objects from the PROB

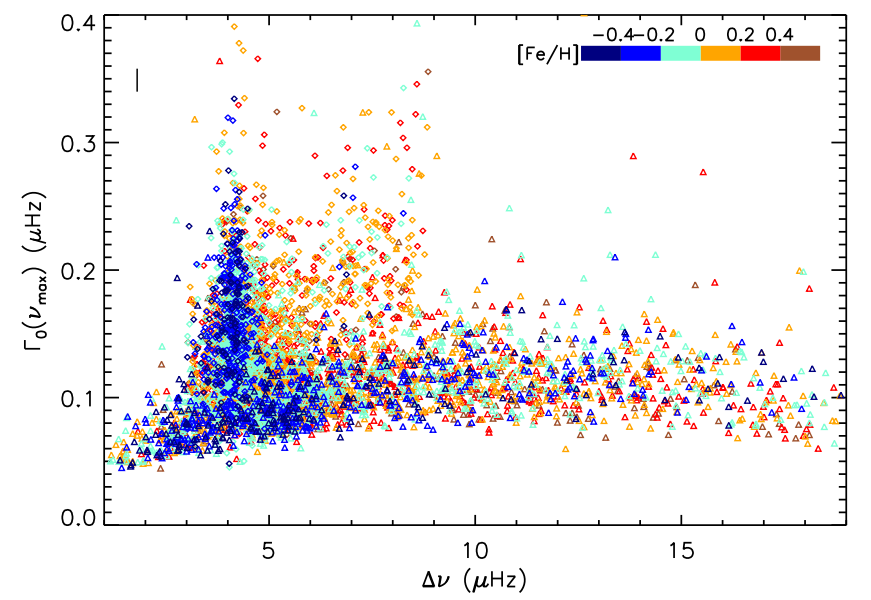

Fig. B.1. Global radial mode width $\left(\Gamma_{0}\left(v_{\max }\right)\right)$ as a function of the large separation $(\Delta v)$ for the PROB method, with the stellar metallicity colorcoded. The diamond and triangle symbols indicate clump and RGB stars, respectively. The mean uncertainty in $\Gamma_{0}\left(v_{\max }\right)$ is given by the vertical black line in the upper left corner.

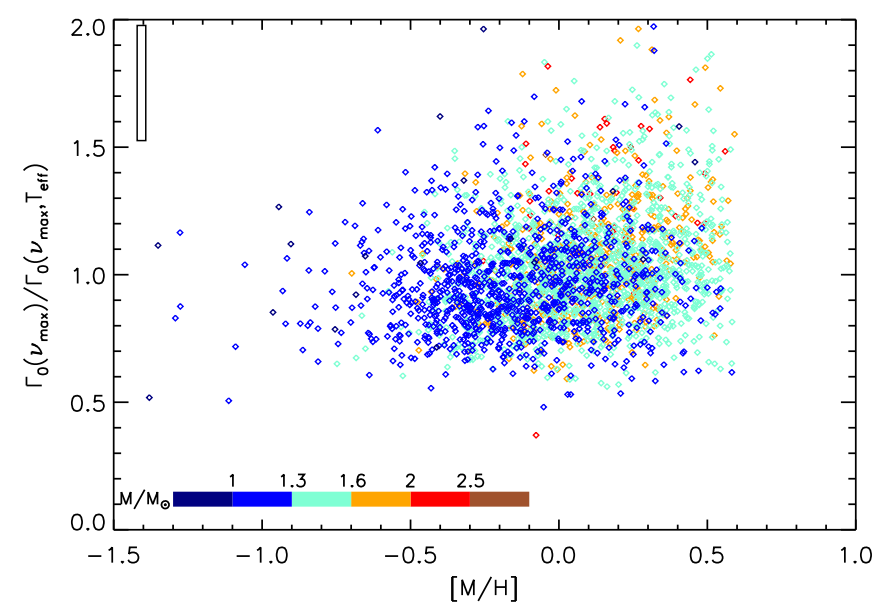

Fig. B.2. Global radial mode width $\left(\Gamma_{0}\left(v_{\max }\right)\right)$ of RGB stars corrected from the $T_{\text {eff }}$ dependence as a function of the metallicity for the PROB method. The mean uncertainty in $\Gamma_{0}\left(v_{\max }\right)$ is given by the black square in the upper left corner.

method. The secondary clump stars exhibit higher metallicity values as expected for younger stars but no other trend is observed for the results from both methods.

Since $\Gamma_{0}\left(v_{\max }\right)$ strongly depend on the $T_{\text {eff }}$ values, the suppression of this dependence is necessary in order to assess the existence of a metallicity influence on the mode width. We therefore use the fits from Sect. 4.2 to correct the mode widths for the influence of $T_{\text {eff }}$ previously characterized in this section. The results, shown in Fig. B.2 for the PROB method, exhibit no significant trend with the metallicity. Therefore, we conclude that we could not highlight a metallicity influence on the mode width with the precision we currently have on this parameter. 